\title{
AN APPLICATION OF THE SEPARATION THEOREM FOR HERMITIAN MATRICES
}

\section{T. L. MARKHAM}

ABSTRACT. Suppose $H$ is an $n \times n$ hermitian matrix over the complex field partitioned as $H=\left(\begin{array}{c}A \\ B *\end{array}\right)$, where $C$ is invertible. Using the separation theorem on eigenvalues of hermitian matrices, bounds are obtained for the eigenvalues of $(H / C)=A-B C^{-1} B^{*}$ in terms of the eigenvalues of $H$ and $C$.

I. Introduction. Suppose $H$ is an hermitian matrix of order $n$ partitioned as

$$
H=\left(\begin{array}{ll}
A & B \\
B^{*} & C
\end{array}\right)
$$

If $C$ is nonsingular, the Schur complement of $C$ in $H$ is $A-B C^{-1} B^{*}=(H / C)$. Haynsworth proved in [2] that the inertia of $H$, denoted $\operatorname{In}(H)$, is $\operatorname{In}(H / C)+$ In $(C)$. The purpose of this paper is to determine bounds for the eigenvalues of $(H / C)$ in terms of the eigenvalues of $H$ and $C$. Our main tool will be the well-known interlacing theorem for hermitian matrices, which we now state for completeness.

Theorem [3]. Suppose $H$ is an $n \times n$ hermitian matrix with eigenvalues $\lambda_{1} \geq \lambda_{2} \geq \cdots \geq \lambda_{n}$. Let $A$ be the principal submatrix of $H$ obtained by deleting the kth row and kth column of $H$. If $\alpha_{1} \geq \cdots \geq \alpha_{n-1}$ are the eigenvalues of $A$, then

$$
\lambda_{1} \geq \alpha_{1} \geq \lambda_{2} \geq \alpha_{2} \geq \cdots \geq \alpha_{n-1} \geq \lambda_{n}
$$

From this classical theorem, it follows easily that if $A$ is a principal submatrix of $H$ of order $p$ with eigenvalues $\alpha_{1} \geq \cdots \geq a_{p}$, then

$$
\lambda_{i} \geq \alpha_{i} \geq \lambda_{n-p+i} \quad \text { for } i=1, \cdots, p \text {. }
$$

Received by the editors December 10, 1973.

AMS (MOS) subject classifications (1970). Primary 15A 18, 15 A57.

Key words and phrases. Separation theorem, hermitian matrices, Schur complement, compound matrix, bounds for eigenvalues. 
With regard to notation, we write $H(i, k, \cdots, n \mid j, k, \cdots, n)$ to denote the minor of $H$ with rows indexed by $(i, k, \cdots, n)$ and columns indexed by $(j, k, \cdots, n)$, where, of course, $1 \leq i, j \leq k-1$. Also, sometimes we find it convenient to denote the eigenvalues of a $p \times p$ hermitian matrix, $M$, by $\lambda_{1}(M) \geq \cdots \geq \lambda_{p}(M)$.

II. Bounds for the eigenvalues of $(H / C)$. Assume $H=\left(\begin{array}{ll}A & B \\ B & B\end{array}\right)$ is hermitian of order $n, A$ is of order $k-1$, and thus $C$ is of order $n-k+1$. Further, suppose $C$ is invertible. Now, if we set $(H / C)=\left(d_{i j}\right)$, then Crabtree and Haynsworth [1] have shown

$$
d_{i j}=\frac{H(i, k, \cdots, n \mid j, k, \cdots, n)}{\operatorname{det}(C)} \text { for } 1 \leq i, j \leq k-1 .
$$

If we let $E=\left(e_{i j}\right)$ where $e_{i j}=H(i, k, \cdots, n \mid j, k, \cdots n)$ for $1 \leq i, j \leq k-1$, then $(1 / \operatorname{det}(C)) \cdot E=(H / C)$. It is easy to verify that $E$ is a principal submatrix of the $(n-k+2)$-compound matrix of $H, C_{n-k+2}(H)$, which is hermitian. Then the eigenvalues of $C_{n-k+2}(H)$, say

$$
\partial_{1} \geq \cdots \geq \partial_{\left(\begin{array}{c}
n \\
n-k+2
\end{array}\right)}
$$

are the $\left(\begin{array}{c}n \\ n-k+2\end{array}\right)$ products $\lambda_{i_{1}} \lambda_{i_{2}} \cdots \lambda_{i_{n-k+2}}$, where $1 \leq i_{1}<i_{2}<\cdots<$ $i_{n-k+2} \leq n$ [4, p. 24], where each $\lambda_{k}$ is an eigenvalue of $H$.

Thus, using (2), we have

$$
\partial_{i} \geq \lambda_{i}(E) \geq \partial\left(\begin{array}{c}
n \\
n-k+2
\end{array}\right)-(k-1)+i \text { for } i=1, \cdots, k-1
$$

Finally, if $\operatorname{det}(C)>0$, we get

(5) $\partial_{i} / \operatorname{det}(C) \geq \lambda_{i}(H / C) \geq \partial_{\left(\left(\begin{array}{c}n \\ n-k+2\end{array}\right)-k+i+1\right)} / \operatorname{det}(C)$ for $i=1, \cdots, k-1$.

We have proved

Theorem 1. Suppose $H=\left(\begin{array}{ll}A & B \\ B^{*} & C\end{array}\right)$ is an hermitian matrix with the dimensions of $A$ and $C$ as specified earlier. Assume $\operatorname{det}(C)>0$, and let $C_{n-k+2}(H)$ denote the $(n-k+2)$-compound matrix of $H$. If we denote the eigenvalues of $C_{n-k+2}(H), C$, and $(H / C)$, respectively, by

$$
\partial_{1} \geq \cdots \geq \partial\left(\begin{array}{c}
n \\
n-k+2
\end{array}\right)
$$


$\alpha_{1} \geq \cdots \geq \alpha_{n-k+1} ;$ and $\beta_{1} \geq \cdots \geq \beta_{k-1}$, then

$$
\frac{\partial_{i}}{\alpha_{1} \cdots \alpha_{n-k+1}} \geq \beta_{i} \geq \frac{\left.\left.\partial_{(} \begin{array}{c}
n \\
n-k+2
\end{array}\right)-k+i+1\right)}{\alpha_{1} \cdots \alpha_{n-k+1}} \text { for } i=1, \cdots, k-1 \text {. }
$$

Clearly, the above result holds a fortiori for $H$ positive definite. In this case, $\lambda_{1} \cdots \lambda_{n-k+2}$ is the largest eigenvalue of $C_{n-k+2}(H)$ and $\lambda_{k-1} \cdots \lambda_{n}$ is the smallest eigenvalue of $C_{n-k+2}(H)$, and we obtain a

Corollary. Under the hypotheses of the theorem with $H$ positive definite, then

$$
\frac{\lambda_{1} \cdots \lambda_{n-k+2}}{\alpha_{1} \cdots \alpha_{n-k+1}} \geq \beta_{i} \geq \frac{\lambda_{k-1} \cdots \lambda_{n}}{\alpha_{1} \cdots \alpha_{n-k+1}} \quad \text { for } i=1,2, \cdots, k-1 \text {. }
$$

We make two simple observations concerning the Corollary. For $k=2$, the Corollary becomes

$$
\frac{\lambda_{1} \cdots \lambda_{n}}{\alpha_{1} \cdots \alpha_{n-1}} \geq \operatorname{det}(H / C) \geq \frac{\lambda_{1} \cdots \lambda_{n}}{a_{1} \cdots \alpha_{n-1}},
$$

which yields $\operatorname{det}(H)=\operatorname{det}(C) \operatorname{det}(H / C)$, a special case of Schur's identity [2, p. 74] since $C$ is of order $n-1$. For $k=3$, the Corollary yields

$$
\operatorname{det}(H / C) / \lambda_{n} \geq \beta_{i} \geq \operatorname{det}(H / C) / \lambda_{1} \text { for } i=1,2 \text {, }
$$

and thus $1 / \lambda_{n} \geq 1 / \beta_{i} \geq 1 / \lambda_{1}$ for $i=1,2$, a reciprocal separation property. Further, we obtain $\lambda_{1}^{2} \geq \beta_{1} \beta_{2} \geq \lambda_{n}^{2}$ from the above in equality.

III. The positive definite case. Suppose $A$ is an $n \times n$ positive definite matrix. Denote by $A_{k}$ the principal submatrix of $A$ contained in rows $1,2, \cdots, k$, for $k=1, \cdots, n-1$, and let $\lambda_{n}(A)$ be the minimal eigenvalue of $A$. As before, $\lambda_{1}(A)$ denotes the maximal eigenvalue of $A$. The following theorem and proof is similar to a result of Watford [5, Theorem 4] on $M$ matrices.

Theorem 2. Suppose $A$ is a positive definite matrix of order $n$. Then

$$
\lambda_{n}(A) \leq \lambda_{n}\left(A \mid A_{1}\right) \leq \cdots \leq \lambda_{n}\left(A \mid A_{n-1}\right),
$$

$$
\lambda_{1}\left(A \mid A_{n-1}\right) \leq \cdots \leq \lambda_{1}\left(A \mid A_{1}\right) \leq \lambda_{1}(A) .
$$

Proof. Assume, first, that $A$ is partitioned as

$$
A=\left(\begin{array}{cc}
B & C \\
C^{*} & D
\end{array}\right)
$$


Now $\lambda_{1}\left(A^{-1}\right)=1 / \lambda_{n}(A)$, and since $(A \mid B)^{-1}$ is a principal submatrix of $A^{-1}$ $[5, \mathrm{p} .251]$, we have $\lambda_{1}\left[(A \mid B)^{-1}\right] \leq \lambda_{1}\left(A^{-1}\right)$, using the separation theorem. Thus it follows that

$$
\lambda_{n}(A) \leq \lambda_{n}(A \mid B)
$$

Next, we note that if $B=A_{p+1}$ in (6), and

$$
A_{p+1}=\left(\begin{array}{cc}
A_{p} & B_{12} \\
B_{12}^{*} & a_{p+1, p+1}
\end{array}\right)
$$

then

$$
\left(A \mid A_{p+1}\right)=\left(\left(A \mid A_{p}\right) \mid\left(A_{p+1} \mid A_{p}\right)\right)
$$

the Haynsworth quotient property [1]. Using (7), we have

$$
\lambda_{n}\left(A \mid A_{p+1}\right) \geq \lambda_{n}\left(A \mid A_{p}\right) \geq \lambda_{n}(A),
$$

and statement (2.1) is immediate. We obtain (2.2) by noting $\lambda_{n}(A)=1 / \lambda_{1}(A)$.

IV. Conclusion. There exist matrices for which the bounds of Theorem 1 are exact. However, even if $H=\left(\begin{array}{ll}A & 0 \\ 0 & C\end{array}\right)$, the bounds may give only rough estimates for the eigenvalues of $(H / C)=A$. For example, if $A=\left(\begin{array}{ll}3 & 1 \\ 1 & 3\end{array}\right)$ and $C=\left(\begin{array}{ll}4 & 3 \\ 3 & 4\end{array}\right)$, then $H=A+C$ has eigenvalues $\lambda_{1}=7, \lambda_{2}=4, \lambda_{3}=2, \lambda_{4}=1$. But $(H / C)=A$ has eigenvalues $\beta_{1}=4, \beta_{2}=2$, and $C_{n-k+2}(H)=C_{3}(H)$ has eigenvalues $\partial_{1}=56, \partial_{2}=14, \partial_{3}=8$, The theorem yields $56 / 7 \geq \beta_{1} \geq$ $14 / 7$ and $14 / 7 \geq \beta_{2} \geq 8 / 7$. It seems likely that one could obtain "tighter" bounds in general by an application of the Courant minimax theorem for hermitian matrices-results which we shall not investigate in this paper.

\section{REFERENCES}

1. D. E. Crabtree and E. V. Haynsworth, An identity for the Schur complement of a matrix, Proc. Amer. Math. Soc. 22 (1969), 364-366. MR 41 \#234.

2. E. V. Haynsworth, Determination of the inertia of a partitioned Hermitian matrix, Linear Algebra and Appl. 1 (1968), 73-81. MR 36 \#6440.

3. Alston A. Householder, The theory of matrices in numerical analysis, Blaisdell, New York, 1964, p. 76. MR 30 \#5475.

4. M. Marcus and H. Minc, A survey of matrix theory and matrix inequalities, Allyn and Bacon, Boston, Mass., 1964. MR 29 \#112.

5. L. J. Watford, Jr., The Schur complement of a generalized M-matrix, Linear Algebra and Appl. 5 (1972), 247-255. MR 46 \#9075. 